\title{
DEMENCIA EN PACIENTES ADULTOS CON SÍNDROME DE INMUNODEFI- CIENCIA ADQUIRIDA, ATENDIDOS EN HOSPITAL MARIO CATARINO RIVAS
}

\section{Dementia in adult patients with acquired immunodeficiency syndrome treated at Hospital Mario Catarino Rivas}

\author{
Arnold Thompson Cerna,* Debra D. Carrillo, ${ }^{* *}$ José A. Pineda, ${ }^{* *}$ \\ Laura I. Coello, ${ }^{* *}$ Isis D. Oseguera, ${ }^{* *}$ Mersys E. Lara, ${ }^{* *}$ \\ Nancy S. Villalobos, ${ }^{* *}$ Rosa E. Zelaya.**
}

\section{RESUMEN}

Introducción: La demencia asociada al virus de inmunodeficiencia humana (D-VIH) es un tipo de demencia subcortical debido a infecciones crónicas por $\mathrm{VIH}$; y combina alteraciones cognitivas, motoras y conductuales, afectando del 20 al 30\% de los pacientes adultos que sufren esta enfermedad. La Escala de Demencia por VIH (EDV) es una herramienta sensible que se utiliza para tamizaje de pacientes infectados por VIH y con riesgo de desarrollar demencia. Objetivos. Aplicar la EDV en pacientes con infección avanzada por $\mathrm{VIH}$ que asistían al Centro de Atención Integral (CAI) del Hospital Mario Catarino Rivas (HMCR) y analizar su relación con el conteo de células TCD4 $<200$. Metodología. Se trata de un estudio cuantitativo, descriptivo, de corte transversal con un muestreo intencionado. En el estudio se incluyó pacientes mayores de 18 años con VIH confirmado, quienes asisten al CAI, alfabetos, con conteo reciente de linfocitos CD4 menor de 200 células y que consintieron participar en el estudio. Se les aplicó la EDV como tamizaje para evaluar su función mental. Resultados. El $81 \%$ de los pacientes entrevistados presentaron riesgo de D-VIH con un rango de edad de

\footnotetext{
* Profesor carrera de medicina. Escuela Universitaria de las Ciencias de la Salud EUCS. Neurologo, HMCR

** Estudiantes de VI año de la Carrera de Medicina, UNAH-VS

Recibido: 18 de Agosto 2,014 Aprobado 20 de Noviembre 2,014
}

mayor prevalencia entre 38 y 57 años, siendo el género femenino el de mayor riesgo. Conclusión de acuerdo a los resultados obtenidos, la EDV es una herramienta costo-efectiva para determinar la función cognitiva en los pacientes.

\section{PALABRAS CLAVE}

Demencia, HIV, Linfocitos T CD4 positivos.

\section{ABSTRACT}

Introduction: human immunodeficiency virus-associated dementia (HIV-D) is a type of subcortical dementia due to chronic HIV infection and combines cognitive, motor and behavioral disorders, affecting 20 to $30 \%$ of adult patients suffering from this disease. The HIV Dementia Scale (EDV) is a sensitive tool to be used for screening of HIV-infected patients and those at risk of developing dementia. Objectives. Apply EDV in patients with advanced HIV infection in patients attending the Honduran Comprehensive Care Center (ISC) and analyze its relationship with CD4 count $<200$ cells. Methodology. This is a quantitative, descriptive, cross-sectional study with purposive sampling. The study included confirmed HIV patients who attend the Mario Catarino Rivas Hospital HMCR, Center Of Integral Attention CAI over 18 alphabets, with recent CD4 lymphocyte count less than 200 cells 
and who consented to participate in the study. EDV was applied as screening to assess their mental function. Results. 81\% of the interviewed patients had HIV risk-D with an age range most prevalent between 38 and 57 years, female gender being the highest risk. Conclusion According to the results, the EDV is a tool to assess cognitive function in patients cost-effective.

\section{KEYWORDS}

Dementia, HIV, CD4-Positive T-Lymphocytes.

\section{INTRODUCCION}

La demencia por VIH es un síndrome que se añadió como un indicador de infección avanzada por VIH en 1987 y fue denominada encefalopatía por el VIH. ${ }^{(1)}$ El complejo de demencia relacionado a la infección avanzada por VIH es una entidad caracterizada por la triada de compromiso cognitivo, síntomas conductuales y motores que generan serias dificultades en la capacidad funcional del paciente. ${ }^{(2)}$

La D-VIH es causada directamente por el $\mathrm{VIH}$ en el sistema nervioso central, pero además por mecanismos indirectos (relación genética de quimocinas-B como la quimio-atrayente de monocitos) generando manifestaciones típicas de demencia subcortical. ${ }^{(3)}$

En el análisis patológico se observa la perdida neuronal, gliosis reactiva y daño en las vainas de mielina. ${ }^{(4)}$

El escenario principal para la vida del VIH lo constituye el receptor de linfocitos CD4 el cual muestra una gran afinidad por las partí- culas proteicas del virus. El receptor de linfocito CD4 es un compuesto proteínico presente en la superficie de monocitos, macrófagos, células dendríticas, linfocitos $\mathrm{T}$ y células gliales. ${ }^{(5)}$

Se han publicado factores de riesgo para el desarrollo de demencia que incluyen niveles altos de ARN del VIH en plasma, conteo de linfocitos CD4 bajo, anemia, género femenino, uso de drogas inyectables, bajo índice de masa corporal, edad avanzada, niveles bajos de hemoglobina y los síntomas constitucionales de la infección avanzada por VIH. ${ }^{(6)}$

El diagnóstico del sindrome de demencia se realiza por exclusión, ${ }^{(7)}$ con síntomas y signos tempranos que suelen ser difíciles de reconocer, por lo que es necesario tener un adecuado plan diagnóstico. Se agrupan los síntomas clínicos en 3 categorías: cognitivos, conductuales y motores. ${ }^{(8-12)}$ Los síntomas cognitivos incluyen compromiso de la memoria de corto plazo, pobre concentración, enlentecimiento mental y pérdida de la flexibilidad y espontaneidad. Cambios de personalidad, apatía, irritabilidad y depresión caracterizan los cambios conductuales. Los síntomas motores pueden ser evidenciados como torpeza o enlentecimiento del motor fino, temblor y debilidad de miembros inferiores, en otros estudios también se demostró que existe un compromiso de la memoria, asi mismo hay enlentecimiento mental, y dificultad de concentración. ${ }^{(7,8)}$

El objetivo del estudio fue, aplicar la EDV en pacientes con infección avanzada por VIH que asistían al CAI del HMCR y analizar su relación con el conteo de células TCD4 $<200$. 


\section{MATERIALES Y METODOS}

Estudio descriptivo de corte transversal con un muestreo intencionado, realizado en colaboración con el personal del CAI del HMCR, quienes a través de los historiales clínicos nos brindaron el resultado del último conteo de CD4 de los pacientes que aceptaron participar en el estudio y se les aplicó la EDV durante el período del 25 al 30 de septiembre del 2011. Los criterios de inclusión fueron edad mayor de 18 años, con infección por VIH confirmada mediante dos pruebas serológicas positivas, conteo reciente de linfocitos CD4 menor de 200, alfabetas que asisten a control en el CAl del Hospital Mario Catarino Rivas y que dieron su consentimiento para participar en el estudio. Se excluyeron del estudio todos los pacientes $\mathrm{VIH}$ negativo, personas menores de 18 años, Conteo de celulas TCD4 $\geq 200$, paciente con retraso mental, antecedentes psiquiátricos, procesos neuroinfecciosos, alcoholismo crónico y adicción a las drogas.

Definición de Caso: Paciente que cumpla los criterios de inclusión, un puntaje menor de 6 en la Escala de Demencia por VIH, lo que corresponde a que el paciente presente: a) déficit de memoria, b) bradipsiquia, c) enlentecimiento de la actividad intelectual, d) alteraciones en la personalidad, e) deterioro en la habilidad de usar conocimiento y f) alteraciones motoras.

Los pacientes en estudio fueron evaluados mediante la aplicación de la EDV la cual ha sido clasificada en cuatro estadios de acuerdo con la gravedad de la afección. El test evalúa los siguientes apartados:

- Registro de Memoria; para su aplicación el paciente inicia pronunciando las cuatro palabras siguientes: perro, sombrero, verde y melocotón donde se le explica al paciente, que en el tercer apartado tendrá que recordar cada una de las palabras.

- Velocidad psicomotora; puntaje máximo de 6 , en este apartado se les solicita que escriban el alfabeto en letras mayúsculas registrando en segundos el tiempo en que lo realizan y se valora con la siguiente puntuación: $\leq 21$ segundos $=6,21-24$ segundos $=5,24-27$ segundos $=4,27-30$ segundos $=3,30-33=2,33-36$ segundos $=1,>36$ segundos $=0$.

- Memoria reciente; puntaje máximo de 4, se pide al paciente que recuerde las cuatro palabras que registro previamente y se otorga un punto a cada palabra correcta.

- Praxis constructiva; puntaje máximo de 2 , se le indica al paciente que copie un cubo previamente dibujado, donde se registra el tiempo y se da la siguiente puntuación: $<25$ segundos $=2 ; 25-35$ segundos $=1$; $>35$ segundos $=0$.

Análisis Estadístico: Se utilizó el programa computarizado de Excel versión 2009.

Consideraciones Éticas: Todos las pacientes aceptaron su participación en la investigación mediante un consentimiento informado, así mismo se obtuvo la aprobación de la Dirección del Centro de Atención Integral (CAI) del Hospital Mario Catarino Rivas y la Escuela Universitaria de Ciencias de la Salud de la Universidad Nacional Autónoma de Honduras en el Valle de Sula. 


\section{RESULTADOS}

De 21 pacientes entrevistados, 17 obtuvieron un valor $<6$ en la escala, lo que representa que tienen un riesgo de demencia por $\mathrm{VIH}$. El riesgo de D-VIH fue mayor en el grupo de población de edad comprendida entre 38 y 57 años, con mayor predominancia en el sexo femenino en el $88 \%$.

\section{DISCUSION}

Los datos obtenidos en esta investigación revelan que la EDS es una herramienta efectiva para la aplicación en personas con alteración de memoria, alteraciones de la praxia o velocidad psicomotora. ${ }^{(8,13)}$ Con relación al aumento del riesgo asociado a mayor edad, corresponde con otros resultados encontrados en otros estudios como la investigación de Valcour, y la cohorte Hawaiana de pacientes con $\mathrm{VIH}-1$, la cual reporta que la demencia por VIH es más frecuente en individuos seropositivos mayores de 50 años de edad, comparado con seropositivos de 20 años. ${ }^{(6)}$ El estudio realizado por Hesse y colaboradores en el Hospital Escuela de Tegucigalpa, Honduras, revelo datos que sugieren que el deterioro cognitivo observado en las personas diagnosticadas con D-VIH mediante el uso de diversas pruebas neuropsicológicas entre ellas la EDV, está relacionado con diversos marcadores inflamatorios indirectos que contribuyen a los cambios patológicos observados en el cerebro de pacientes con este síndrome clínico. ${ }^{(3)}$

Recientemente se ha planteado que gracias al avance en la terapia antirretroviral, los casos de D-VIH en etapa avanzada han descendido pero esto plantea un reto cada vez mayor en desarrollar herramientas para que esta entidad clínica sea reconocida cada vez más de forma temprana. ${ }^{(14)} \mathrm{El}$ mayor reto para el futuro es el desarrollo de marcadores que permitan el diagnóstico temprano a través de estudios de líquido cefalorraquídeo o de estudios de neuro imagen. ${ }^{(15)}$

En conclusión, de acuerdo a los resultados obtenidos, la EDV es una herramienta costo-efectiva para determinar la función cognitiva en los pacientes.

\section{BIBLIOGRAFÍA}

1. Marder K, Albert SM, Mc Dermoth MP et al. Inter-rater reliability of a clinical staging of HIV-associated cognitive impairment. Neurology. 2003; 60:146773.

2. Sacktor NC, Wong M, Nakasujja N, Skolasky RL, Selnes OA, Musisi S, et al. The International HIV Dementia Scale: a new rapid screening test for HIV demen- tia. AIDS. 2005;19:1367-1374

3. Hesse H, Medina MT, Bu-Figueroa E. Caracterización clínica y neuropsicológica de pacientes Hondureños con demencia asociada al VIH-1 y su correlación con las moléculas inflamatorias en LCR y suero. Rev. Med post UNAH 2003; 8 (1): 45-56. 
4. Kipnis J, Derecki NC, Yang C, Scrable $\mathrm{H}$. Immunity and cognition: what do age-related dementia, HIV-dementia And 'chemo-brain' have in common? Trends immunol 2008; 29: 455-463.

5. Ott A, Breteler MM, van Harskamp Claus JJ, van der Cammen TJM, Grobbee DE, Hofman A. Prevalence of Alzheimer's disease and vascular dementia: association with education. The Rotterdam study. BMJ 1995; 310:970-973.

6. Prencipe $M$, Casini AR, Ferretti C, Lattanzio MT, Fiorelli M, Culasso F. Prevalence of dementia in an elderly rural population: effects of age, sex, and education. Journal Neurology NeurosurgeryPsychiatry 1996; 60:628-633.

7. Kaul M, Lipton SA. Mechanisms of neuroimmunity and neurodegeneration associated with HIV-1 infection and AIDS Neuroimmune Pharmacol.2006; 1:138-151.

8. Wind AW, Schellevis F, Van Staveren G, Scholten RJPM, Hooijer C, Jonker C, et al. Limitation of the Mini-Mental State Examination in diagnosing dementia in general practice. Int J Geriatr Psychiatr 1997; 12:101-108.

9. Robinson-Papp J, Byrd D, Mindt MR, et al. Motor function and human immunodeficiency virus-associated cognitive impairment in a highly active antiretrovi- ral therapy-era cohort. Arch Neurol 2008; 65:1096-101.

10. McArthur J C, Houghey N, Garther S, Human, et al. Inmunodeficiency Virus associated dementia: An evolving disease. Journal of Neurovirology 2003; 9:205-221.

11. Barahona Mejía A, Reyes Ticas A. Trastorno neurológicos y Psiquiátricos asociados al Síndrome de Inmunodeficiencia Adquirida en pacientes hospitalizados en el Hospital Escuela de Tegucigalpa, Honduras. Rev. Med Hond 1999; 67: 243-246.

12. Beard M C, Kokmen E, Offord K P, Kurland $\mathrm{L}$ T. Lack of association between Alzheimer's disease and education, occupation, marital status, or living arrangement. Neurology 1992; 42:20632068.

13. Power C, Selnes O, Grim J, Mcarthur J. The HIV dementia scale: a rapid screening test. J aids. 1995; 8:273-8.

14. Sacktor NC. Evolving clinical phenotypes in HIV-associated neurocognitive disorders. Curr Opin HIV AIDS 2014; 9:517-20.

15. Chan P. HIV associated neurocognitive disorders in the modern antiviral treatment era: prevalence, characteristics, biomarkers and effect of treatment. Curr HIVIAIDS Rep 2014; 11:317-24. 